\title{
Follower-Leader Concept in Microscopic Analysis of Pedestrian Movement in a Crowd
}

\author{
Jana Vacková ${ }^{1}$, Marek Bukáček ${ }^{2}$ \\ ${ }^{1,2}$ FNSPE, Czech Technical University in Prague \\ Břehová 7, Prague, Czech Republic \\ janca.vackova@fjfi.cvut.cz; marek.bukacek@fjfi.cvut.cz
}

\begin{abstract}
This paper presents a microscopic analysis of factors influencing pedestrian movement and interactions with their surroundings for two considered modes: independent movement influenced only by the surrounding conditions and synchronized movement based on following another pedestrian. This study analyses which of these effects prevail in different phases of the movement. The results show that the significant value of correlation between pedestrian velocity and corresponding individual density is observed mainly during approaching the crowd. Contrarily, in the segment of pedestrian trajectory which corresponds to movement inside the crowd, correlation between the velocity of a follower and a leader is more important. This confirms that the pedestrian behaviour in a crowd is a complex field.
\end{abstract}

Keywords: pedestrian dynamics, egress experiment, correlation, individual density, follow-the-leader.

\section{Introduction}

The prediction of a pedestrian's velocity is one of the most crucial tasks for evacuation and egress modelling. Naturally, pedestrians react to the conditions in their surroundings and interact with others. Velocity-density dependency represents one of the most popular diagrams in this field [4]. Nevertheless, having discussed in [2], the shape of this relation significantly differs with the phase of the motion.

Social interaction may be categorized as social interactions between friends, colleagues or family members and the crowd interaction that is not driven by any prior intention, but only the temporal purpose. In this paper, we put aside the social level and focus only to individuals. The concept of correlations between velocities of the nearest neighbors in a specific angle was studied in [5], the results were interpreted on group level. In this study, all conclusions will be provided on individual level.

Similarly to [2], the Pearson correlation coefficient $R\left(\rho_{\omega_{\alpha}}, v_{\alpha}\right)$ is used to measure the strength of a pedestrian's $\alpha$ reaction expressed by their velocity $v_{\alpha}$ to their surroundings described by an individual density $\rho_{\omega_{\alpha}}$. Rolling window with memory time $\tau=1.56 \mathrm{~s}$ enables to watch a progress in time. Moreover, the same technique visualizes the correlation $R\left(v_{\alpha}, v_{\beta}\right)$ which expresses the relation of a pedestrian and their nearest predecessor ('leader') $\beta$.

This study is based on the egress experiment summarized in [1]. Pedestrians randomly entered the room by one of three entrances, walked to the opposite wall and left the room by one exit. By controlling input flow, different conditions from free flow to congestion in the exit area were achieved.

\section{Pedestrian velocity, individual density and correlation}

The velocity of a pedestrian is calculated as usual using central differences of space coordinates. There are many ways to enumerate the density, in details [3]. We use the concept introduced in [2], where each pedestrian $\alpha$ contributes by their individual density distribution $\mathrm{p}_{\alpha}(\mathbf{x})$ to the density distribution for the whole area $\mathrm{p}(\mathbf{x})$. In other words, every single pedestrian is considered to be a source of density distribution (a cone was picked as the best shape).

Fig. 1, Left shows the trend of these variables for one chosen trajectory. The trend of their velocity and the individual density indicates short free flow phase followed by gradual joining a crowd 
characteristic by the decrease in velocity with increasing density. These trends dramatically changed since the pedestrian fully entered the crowd - their velocity reaches almost zero and starts growing as the pedestrian adapts to new conditions.
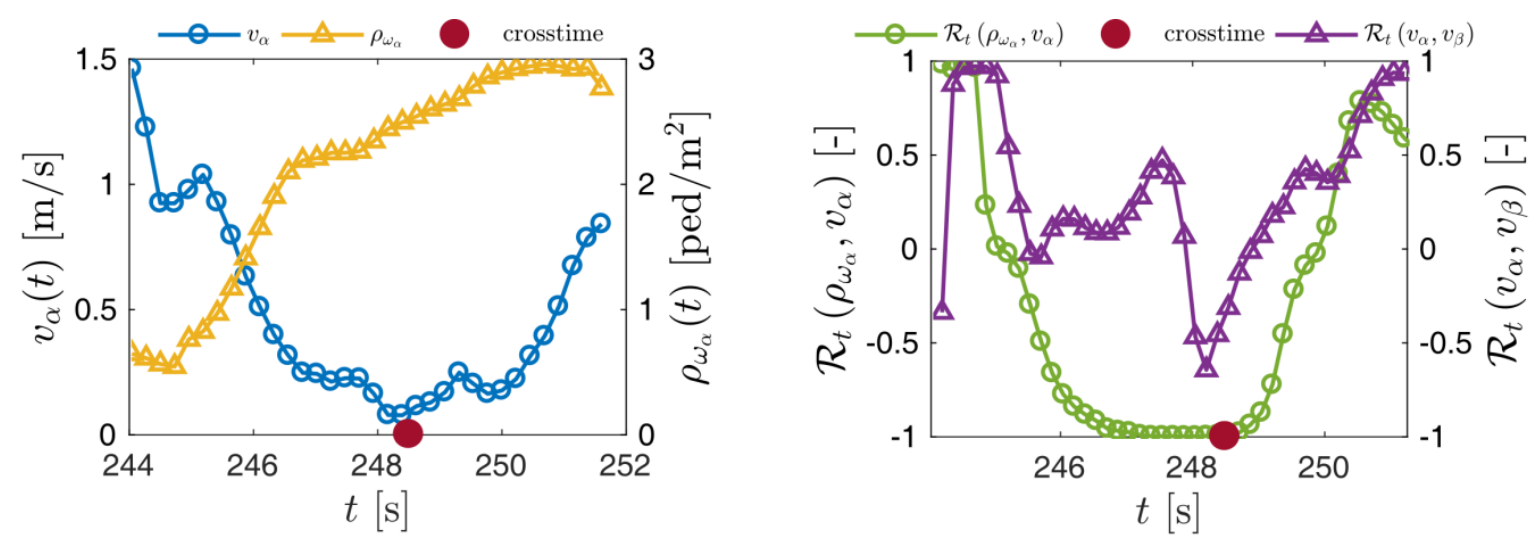

Fig. 1: Left: Velocity (blue circles) and individual density (yellow triangles) for pedestrian $\alpha$. The time of the approach of pedestrian $\beta$ to the pedestrian $\alpha$ at a distance of $0.75 \mathrm{~m}$ (crosstime) is visualized by the red point. Right: The correlation of density and velocity for pedestrian $\alpha$ (green circles), the correlation of velocities for pedestrians $\alpha$ and leader $\beta$.

The behaviour of the pedestrian in free flow is not quite controlled by either local density or response to a certain leader (Fig. 1, Right). The positive correlation of velocity and density indicates competitive behaviour when the occurrence of another pedestrian motivates participants to move faster. Moreover, the positive correlation between the velocities for pedestrians $\alpha$ and $\beta$ indicates that both are affected by the same environmental effects.

While approaching and joining the crowd, velocity of any pedestrian is strongly related to their individual density - significant negative correlation is observed in Fig. 2, Left, where curves are plotted at the normalized time $t_{N}$ (for each pedestrian $\mathrm{t}_{\mathrm{N}}=0$ and $\mathrm{t}_{\mathrm{N}}=1$ corresponds to the entry and exit time, respectively). The joining the crowd phase was observed for $t_{N}$ between 0.2 and 0.6.

Although this correlation is sensitive to all possible deviations, it is obvious that the most paths were fully joined the crowd in $t_{N}$ from 0.5 to 0.7 and then the expected negative correlation completely turned positive - despite the increasing individual density, the velocity is slowly growing. An explanation of this phenomenon including the flow conservation law is described in [2].

As this phenomenon represents just a coincidence, not causality, there is no prediction power of such dependence. The model of increasing velocity based just on increasing density is not applicable; it is necessary to look at this particular part of movement in a detail.
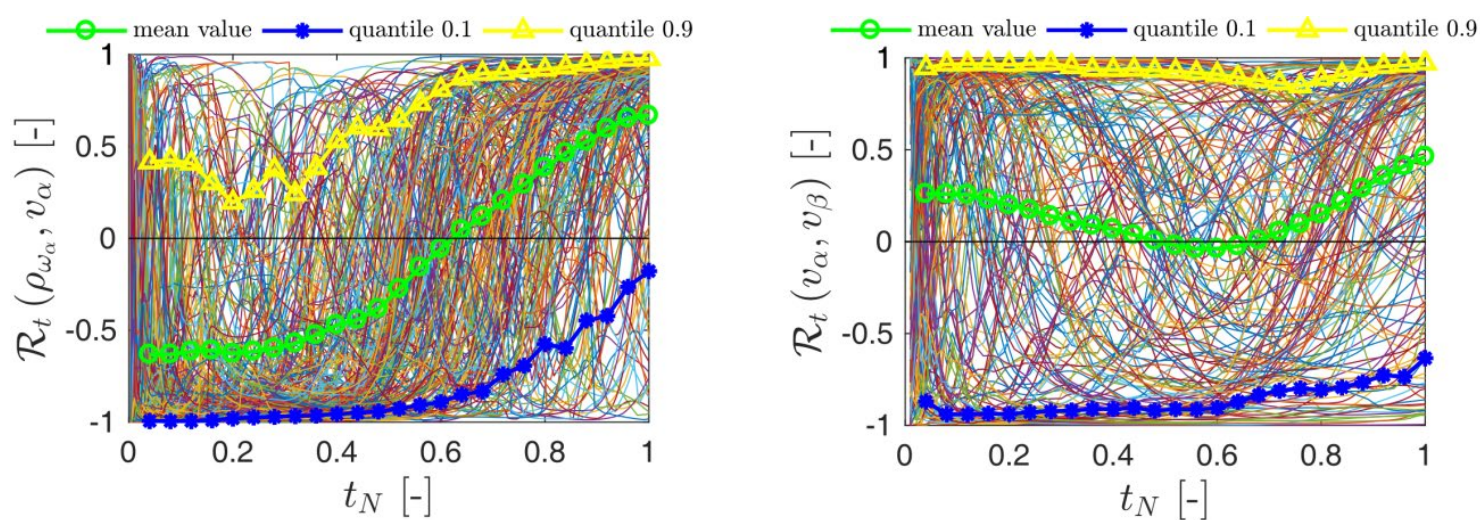

Fig. 2: Correlation between individual density and velocity (Left) and between follower and leader velocities (Right) - for each pedestrian corresponding to travel time $\in(\mathbf{7}, \mathbf{1 5}) \mathbf{s}$ and mean occupancy $\in(\mathbf{7}, \mathbf{1 5})$ ped. Mean value (green line with circles), lower (blue line with stars) and upper (yellow line with triangles) decile are visualized. All plotted with respect to normalized time $\boldsymbol{t}_{\boldsymbol{N}}$. 


\section{Follower-leader concept}

The studied crowd at the exit area has an internal structure similar to a multi-row chaotic queue; therefore the pedestrian standing behind someone else often just copies the movement of a predecessor. To explain this behaviour, simple follow-leader model inspired by vehicular traffic might be developed.

The intended algorithm selects suitable candidates to be a leader for each pedestrian with respect to their distance and direction. As a result of that, the movement of so-called follower might be modelled (predicted) using information of the movement of their leader.

In this proof-of-concept study, the pedestrian $\beta$ is defined as a leader for pedestrian $\alpha$ from the point in time when $\alpha$ approaches $\beta$ at a distance $0.75 \mathrm{~m}$ according to our assumption that $\beta$ is closer to the exit than $\alpha$. As shown in Fig. 1, Right, the correlation of such leader's and follower's velocity increases shortly after crosstime, and it is followed by the mentioned changes in the trend of velocity-density correlation. Furthermore, this phenomenon seems to be global - the Fig. 2, Right demonstrates that the mean correlation of leader's and follower's velocities significantly grows in the crowd part of the egress process.

It is worth noting that a leader for one pedestrian can be at the same point in time a follower for another, and, although the leader and the follower have the same goal, there does not have to be any psychological link between them. This approach is not related to controlled evacuation models, where leaders are often defined in advance and have a special role during an evacuation.

However, no behaviour pattern is universal for all pedestrians. Similar to heterogeneity in velocity, aggressiveness or path selection, heterogeneity in the behaviour inside crowd might be introduced as well.

\section{Conclusions}

This article investigates the correlation of pedestrian velocity and individual density during the whole process of passing a room. As shown, the expected negative velocity-density correlation holds only in the phase of approaching the crowd. To predict the velocity of an individual, we introduced the follow-leader concept to replace a velocity-density dependency by leader-follower velocity dependency. The definition of a leader is individual and based just on the position in the room and the velocity that enables automatic detection. The provided correlation study proved the competency of such an approach that can be formulated as movement model on microscopic scale.

\section{Acknowledgements}

Research presented in this work has been supported by the Grant SGS18/188/OHK4/3T/14 provided by the Ministry of Education, Youth, and Sports of the Czech Republic (MŠMT ČR).

We would like to thank GAMS team members, namely Pavel Hrabák and Matěj Kotrba, for significant help with organizing the experiment. All experiment participants approve for the experimental data to be stored, used and published for academic purposes.

For more details see our webpage http://gams.fjfi.cvut.cz/topics/pedstrian-dynamics/.

\section{References}

[1] M. Bukáček and P. Hrabák and M. Krbálek, "Microscopic Travel Time Analysis of Bottleneck Experiments," Transportmetrica A: Transport Science, 2018.

[2] M. Bukáček and J. Vacková, "Evaluation of pedestrian density distribution with respect to the velocity response," in Traffic and Granular Flow, 2017, submitted.

[3] B. Steffen and A. Seyfried, "Methods for Measuring Pedestrian Density: Flow, Speed and Direction with Minimal Scatter," Physica A 389(9): 1902 -- 1910, Elsevier, 2010.

[4] A. Schadschneider and D. Chowdhury and K. Nishinari, Stochastic Transport in Complex Systems. Elsevier, 2010.

[5] J. Poryzcki and J. Was and L. Hedayatifar and F. Hassanibesheli and K. Kulakowski, "Velocity correlations and spatial dependencies between neighbors in a unidirectional flow of pedestrians," Physical review E, 2017. 